\title{
ACUTE MYELITIS PRECEDED BY ACUTE OPTIC NEURITIS.'
}

By J. 'T. ESKRIDGE, M. D, DENver, CuI..

1 Presented to the American Neurological Association at the Annual Meeting in Philadelphia, June, 1890.

\footnotetext{
THE subjoined case of myelitis is of interest chiefly on 1 account of the rarity of the complication, or rather, the first observed neural disturbance, acute optic neuritis.
}

Charles B., aged forty-nine, engaged in railroad bridge building the last few years of his life, but formerly a stage driver in the mountain towns of Colorado, gave history of syphilitic infection about twenty years ago. He stated that there were two or three sores on the penis at the time, and that no constitutional symptoms followed. With the exception of the venereal trouble, he had always been in apparently perfect health until the beginning of his fatal illness. He had always indulged in alcohol freely, taking on an average, four to six drinks of whiskey daily, when he was where he could obtain them, but rarely drank to the point of intoxication.

On Jan. I, I889, he was feeling quite well, but noticed that after he had been looking at the sun's eclipse a short time, the vision of the left eye seemed defective. The next day there was a dull, heavy pain in this eye and it was increased by bright light. He did not seek medical advice at this time, but continued his work at bridge building. Two or three days later his vision became so poor in both eyes that he was compelled to discontinue his work. One week from the time that he had first noticed defective vision in the left eye he was totally blind in both eyes. At this time he consulted the railroad surgeon, Dr. Lay, of Denver, who has kindly given me many points in the history and to whom I am indebted for the privilege of studying and publishing the case.

Jan. 7 th, when he first came under Dr. Lay's care, he he was seen by an oculist, Dr. E. C. Rivers, who found optic neuritis with white atrophy; vision in each eye, $=0$. 
There did not seem to be light perception. The next day (Jan. 8th), the right leg was observed to be weak and used awkwardly, but he walked from his boarding-house to the office of Dr. Lay and back, a distance of several blocks. On the following day (Jan. 9th), he was unable to leave his bed, as both legs were paretic. A few days later both legs were completely paralyzed, and about this time the sphincters of the bladder and anus began to be affected, and were soon completely paralyzed. Up to this time (about Jan. I 5 th), he had suffered no pain and had experienced no girdle sensation around the body. About Jan. I 8 th, he began to complain of a sensation as if there was a weight constantly lying on his chest, and found some difficulty in breathing. About Jan. 22d the arms became paretic, and he suffered pain, severe and shooting in character, in arms and in lower cervical and upper dorsal regions of the spine. The above history is as accurate as I was able to obtain it, as no records had been kept of the case prior to my seeing it.

On Jan. 26th, I was asked by Dr. Lay to see the patient in consultation with him. I learned from the patient that the night previous to my visit he had been able to see a bright light for the first time in more than two weeks. I inquired if he had ever been struck on the back, if his spine had been injured in any way; or if he had been subject to undue exposure, or lifting heavy weights previous to the onset of his trouble. His answers to my questions were all in the negative. No apparent cause, except possibly syphilis, was found for the myelitis.

Examination, Jan, 26, 1889:

The legs were swollen and incapable of the slightest voluntary motion, all their muscles thoroughly flaccid. and the skin over them cool and death-like. Arms and hands retained but slight power of voluntary motion, the hands less than the arms, and the latter less than the shoulder muscles. The ulnar sides of the hands and fore-arms were weaker than the radials. Each hand registered, with the dynamometer, 3. All the abdominal muscles were paralyzed. The lower portion of the chest moved but little during respiration. No affection of the muscles of the facc, tongue, pharynx or larynx. The sphincters of the bladder and anus completely paralyzed. All the reflexes, deep and superficial, in the trunk and extremities were absent. No response to the faradic current was obtained in the legs or arms. No galvanic battery was at hand, and consequently, no tests for reactions of degeneration were made.

In the legs, and in the trunk, as far up as the sixth intercostal space, all sensation was abolished. 
He did not recogni\%e the prick of a pin, the contact of hot and cold substances, the position of his legs, nor could he tell when the legs were moved. No sensation was felt when the catheter was passed into the bladder. On the chest, latterly and anteriorly, from the sixth to the third intercostal space he recognized the prick of a pin as painful, but could not feel the contact of smooth substances. Hot and cold substances were not distinguished as such, but were felt as painful, the cold causing the greater pain. From the third intercostal space upward, sensation was nearly normal, except in the second intercostal space, over which there was some hyperesthesia. He was so weak that every movement of the cliest and arms gave him such intense pain that I refrained from testing sensation over the back. The arms were (and have been for several clays) the seat of a great deal of pain, sometimes dull, but usually sharp and lancinating in character. The pain prevented slecp unless it was relieved by morphia. The arms were not tender to the touch. There was considerable twitching of the muscles of the upper extremities. Movements of the arms, both voluntary and passive, increased the pain in the lower cervical and upper dorsal regions, and in the arms. The sense of touch was nearly or quite lost in the arms and hands, and with them he was unable to distinguish hot and cold substances as such. Ice or moderately cold water, he said, pained him, but dicl not feel cold. He felt the contact of hot cloths and warm metals, but said they did not feel warm, but gave a pleasant sensation. He felt the prick of a pin in the hands and arms, and could locate the position of the sharp points of the asthesiometer, but he was unable to tell the position of either hand or arm. When I moved the hands or arms he could not tell in what direction I was carrying them, nor which arm I was moving. A large bedsore had formed over the sacrum and was increasing in size, despite the utmost cleanliness of the parts. Wherever the skin over the legs was subjected to pressure, sores had begun to form. He lad scratched himsclf over the breast opposite the fifth and sixth intercostal spaces, and the irritated parts had become considerably inflamed and showed no tendency to heal.

Special Senscs.-Eyes : Light perception, with slight iritic reflex, was seen with each eye in the presence of a strong light. No opthalmoscopic examination was made at the time on account of the extreme fcebleness of the patient. Hearing, taste, and smell were preserved and about normal. The mind was perfectly clear and he had no head- 
ache, and had complained of none since the beginning of the spinal trouble. Respirations were slowed at that time from the effects of morphia, under the influences of which it was necessary to keep him on account of pain. Pulse was IIO; temperature, $99^{\circ}$. He continued to get weaker, and died about one week later (Feb. 3 ).

During the last week of his illness, the morning temperature varied from $98^{\circ}$ to 99.5 . Once it registered $103.2^{\circ}$. The evening temperature varied from $99.2^{\circ}$ to $102^{\circ}$. The usual evening temperature was $100^{\circ}$ or $101^{\circ}$. The day before, and the day of his death, it registered $103.2^{\circ}$, probably septic in character from the foul and sloughing bedsores. The pulse varied from 100 to 140 per minute, and the rcspirations from 22 to 46 .

Sectio-Cadavaris sixteen hours after death, by the writer, assisted by Drs. Fisk, I,yman and Weist.

The bed-sore over the sacrum was six inches in diameter, with ragged, sloughing edges, and at the centre extended down to bone, but no opening into the spinal canal was found. Small prcssure sores existed over the buttocks, thighs, calves, and heels.

Cord.-The dura presented nearly a normal appearance, except opposite the sixth and seventh cervical and first dorsal vertebra, where it was thickened and inflamed. No pus was found except in two or three spots, each about onc-quarter of an inch in diameter, on the outer surface of the mcmbrane, opposite the sixth cervical vertebra. No bone discase was discovered. The pia was but slightly injected and did not seem abnormally adherent to the cord. Thc latter appeared slightly swollen, but to the touch was of nearly normal consistence. On section the cut surfaces were vascular and white, and gray matter were not clearly dcfined,

Braill.-The membranes and brain substance appeared normal throughout to careful macroscopic examination. The cord and its membranes, the optic nerves, chiasm and tracts, the great ganglia, the median portion of each occipital lobe, and the pons and medulla were placed in Muller's fluid.

Mirroscopical Examination.-The cord was fairly well 
hardened, but the sections from the brain remained in the fluid too long and became granular. Stained sections from the lumbar, dorsal, and lower cervical regions of the cord showed marked inflammatory changes. The changes were diffused throughout nearly the entire cord substance, and not limited to certain portions of the cord, as we find in many cases of myelitis. In the upper cervical region and in the medulla the parts presented a normal appearance. Numerous micropic sections were made from portions of the brain, preserved in Müller's fluid, but the parts had become so granular that no satisfactory conclusion could be reached in regard to the existence of patches of intracerbral inflammation to account for the blindness.

Remarks.-From the paucity of literature on the subject, as well as from the writer's personal experience, the association of optic neuritis with myelitis is extremely infrequent.

Henry D. Noyes, M. D., in his "Treatise on Diseases of the Eye" (edition of I884, p. 3II), says: "Acute myelitis has, within a few years, been accompanied by affections of sight. The first case was published by Steffen and Erb, another by Dr. Seguin, of this city (New York), and I have joined Dr. Seguin in contributing another. The eye symptoms are those of acute, but moderate, neuritis optica, with remarkable impairment of the visual field and of central vision. There may be entire loss of direct sight, there may be any kind of irregularity in the fields, including total abolition on both sides or affection of one only ; there may be repeated recoveries of sight and relapses. The peculiarity of the cases has been that vision, both direct and indirect, should undergo such great and unexpected variations. The lesion of the cord was in its lower and middle portions, as was fully manifested by symptoms of the bladder and the lower limbs. No explanation of the optic neuritis has been offered, although we may bear in mind that a root of the tractus has been traced by Stirling into the crus cerebri at the red nucleus of the 'hood.' All the cases have gotten well, both in respect to sight and to the functions of the cord. In my own case, large doses of iodide of potassium 
were employed, gradually reaching three hundred grains daily, and were well bornc. The case occupied about four months in its evolution."

Seguin, in his Opra Minora, in an article entitled: "On the Coincidence of Optic Neuritis and Subacute Transverse Myelitis," discusses at some length, all the cases referred to. by Noyes, and concludes as follows :

"In the spinal cord the inflammatory changes were in the clorsal region in all the cases, but in all other respects there were marked differences.

"In Case I., the right half of the spinal cord no doubt contained most of the lesions.

"In Case II., the asthesodic region of the cord (posterior gray matter or peri-ependymal region?) was chiefly involved.

"In Case III., the entire structure of the cord must have been slightly affected, the motor region most. The comparative escape of the bladder in Case III. (no retention), is instructive anatomically, as the limitation of the numbness to the altitude of the region of the groin would indicate that the lesion was in the lowest dorsal or upper lumbar region of the cord, below the vesical centre. In Cases I. and III., where the limits of numbness and the constriction band indicated disease of the mid-dorsal portion of the cord, retention and cystitis occurred.

"The question naturally arises : Is there any causal or physiological relation between the two sets of phenomena observed in these three cases?

"Prof. Erb answers in the negative, and it seems to me that with our present knowledge of the relations between the optic apparatus and the spinal cord we must, in agreement with him, consider this association of optic neuritis and transverse myelitis as accidental."

Gowers, in his Manual of Diseases of the Nervous System, states: "In rare cases of myelitis, optic neuritis has been observed, without any intracranial complication to cause it." In a foot note he refers to a case, reported by Sharkey and Lawford (Trans-Ophth. Soc. 1883), and to one, observed by Dr. Dreschfield, of optic neuritis and dissem- 
inated myelitis. The latter case terminated fatally. Whether or not the formerdicl, is not so stated directly, but one naturally infers such a termination from the character of his reference to the case. Gowers remarks: "1t is probably not the result of inflammation of the spinal cord, but is an associated and similar lesion, the result of the cause of the inyelitis. It is noteworthy that most of the cases thus accompanied have been instances of disseminated myelitis, a form that suggests a cause acting widely on the nervous system. In the case of Sharkey and Lawford the optic neuritis reached its height some weeks before the occurrence of the first spinal symptoms, and in the cord were two separate and distinct foci of inflammation."

The case, a detailed report of which is found in this paper, differs from the one reported by Erb, and from the two described by Seguin in his Opra Minora, in that it was a severe case of acute myelitis terminating fatally in five weeks from the first appearance of eye-symptoms, and that the optic neuritis was decided and well-marked when first seen, no vision. except slight light perception, returning subsequently. It differs from those cases referred to by Gowers, as observed by Sharkey and Lawford, and by Dreschfield, in that there was diffuse ascending myelitis and not disseminated as those cases seem to have been.

There is one curious noteworthy fact observed in several of the reported cases, as well as in the one which forms the subject of this communication, viz: the myelitis began in the lower portion of the cord. In the writer's case the inflammation began in the extreme lower portion and gradually travelled upward. In Dreschfield's case, and in the one under discussion, the optic neuritis reached its height before any cord symptoms were manifest, in the former the interval being two weeks, in the latter case, a few days.

It is useless to speculate, in the absence of further observations, as to the cause of optic neuritis in association with acute myelitis. The writer can only regret that through his negligence he allowed the specimens taken trom the brain to remain in Müller's fluid so long that they were unfit for reliable microscopical examination. 